\title{
44568 - ANTERIOR MEDIASTINITIS FOLLOWING TRAUMATIC LARYNGEAL MASK PLACEMENT
}

\author{
Chidam Yegappan, Memorial University of Newfoundland, St. John's, NL, Canada; \\ Edwin Redmond, Memorial University of Newfoundland; \\ Kenneth LeDez, Memorial University of Newfoundland;
}

PURPOSE: To describe a case of a laryngeal tear following traumatic placement of a laryngeal mask (LMA) in a patient with a difficult airway.

CLINICAL FEATURES: Consent was obtained in accordance with local institutional guidelines prior to submission of this case report.

A 60 year old, $80 \mathrm{~kg}$ male, ASA II, presented to a peripheral hospital for an elective excision of an epididymal cyst and hydrocele repair under general anesthesia. He had no prior surgery or anesthesia. Medical history included hypercholesterolemia, dermatitis herpetiformis and glaucoma, for which he was on fenofibrate, dapsone and timoptic drops respectively. Examination of the airway revealed a short muscular neck with a Mallampati class 3 view. The patient was induced with 100mcg Fentanyl, 200mg Propofol, and 200mg Succinylcholine and a size 4 LMA was place with difficulty. Anesthesia was maintained with $2 \%$ Sevofluorane, and a 50/50 oxygen/nitrous oxide mixture with the patient breathing spontaneously. The surgery and recovery room stay were uneventful and he was discharged home postoperatively.

Two days later, the patient returned to the local emergency department with increasing shortness of breath and right upper chest discomfort. Chest x-ray showed right basal atelectasis and a paratracheal soft tissue density. pO2 was $75 \mathrm{mmHg}$ on a room air arterial blood gas. An urgent CT Pulmonary Angiograph (to rule out pulmonary embolism) showed extensive mediastinitis. Subsequently, the patient was placed on $40 \%$ oxygen and transferred to our tertiary care center for a bronchoscopy and right sided thoracotomy to drain the mediastinal abscesses.

An initial awake look with topical anesthesia was followed by rapid sequence induction with 100mg Succinylcholine and 300mg Propofol. A Glide scope ${ }^{\mathrm{TM}}$, used for intubation, provided a grade I view of the larynx and revealed a small necrotic area just proximal to the epiglottis, presumably consequent to the LMA placement and the source of the mediastinitis. Following thoracotomy, the patient was transferred to the ICU intubated and ventilated. He had a complicated course requiring Xygris and vasopressors for septic shock; however, he gradually improved and was extubated approximately 3 weeks after thoracotomy and discharged home one month post-op. He returned 2 weeks after discharge for CT guided drainage of a recurrent mediastinal fluid collection but was otherwise doing well.

CONCLUSION: Complications associated with the use of LMAs are rare; in fact, we could only find one case fatality reported in the adult literature (1). There, the use of an intubating LMA resulted in an esophageal perforation while an endotracheal tube was passed through the mask. We report a case of laryngeal trauma secondary to LMA placement leading to mediastinitis. This illustrates that even with less invasive airway tools, traumatic placement can lead to unfortunate complications.

REFERENCES: 1. Anes. 1999 54: 166-171. 